\title{
Drag Estimation on Wedge-Shaped Protuberances in High-Speed Flows
}

\author{
Abhinav Kshitij \\ Arizona State University, Tempe, AZ, 85287 \\ Simon Prince and John L. Stollery \\ Applied Aerodynamics Group, Cranfield University, Cranfield, UK
}

\begin{abstract}
A semi-empirical method is developed to estimate drag on wedge-shaped projections in hypersonic flow. Force balance measurements from gun tunnel tests directly measure total drag on blunt wedges, where the boundary layer and the entropy layer are weakly coupled. Detailed flowfield analysis from numerical simulations provides estimated locations of peak pressure ratio and skin friction. Schlieren images are used for detecting incipient separation in incoming flows with laminar and turbulent boundary layers. Test results indicate presence of local hotspots at reattachment points of strong detached shocks on wedge compression ramp, and of primary and secondary vortical structures around lateral faces. Total drag is found to decrease with decreasing bluntness but increasing slenderness in wedges tend to increase skin friction drag.
\end{abstract}

\section{Nomenclature}

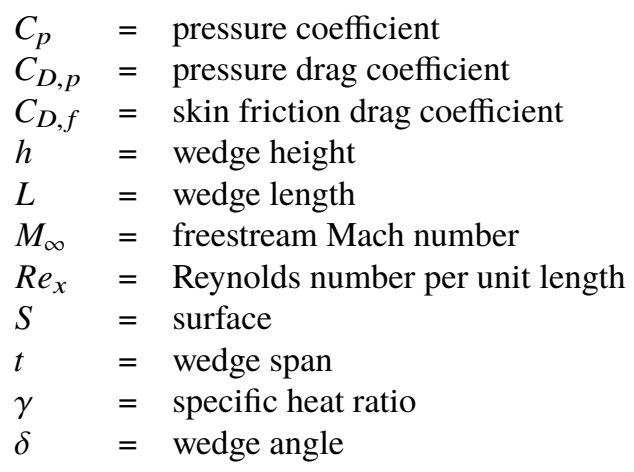

\section{Introduction}

\begin{abstract}
ERODYNAMIC fairings are used on the external surface of rocket and missile fuselages to protect sensitive equipment A and structure. This is particularly important for the high speed flight that experiences high heating rates and aerodynamic forces. Each of these fairings will contribute to the overall vehicle drag force. And together they represent a significant drag increment over the equivalent smooth surface body. This is particularly important in high speed flight that experiences high heating rates and drag force.

This paper presents an experimental and computational study aimed at identifying a simple theoretical approach to predicting drag increment for wedge-like fairings which will allow design engineers to quickly and accurately determine the full configuration drag by calculating the drag of the smooth surface baseline geometry and then adding the accumulated drag increment, without the need to resolve each fairing in the geometry.

This study has been performed for a family of wedge fairing configurations for the cases of laminar and turbulent incoming boundary layers at a constant Mach number of 8.2 and a Reynolds number of $9.0 \times 10^{6} / \mathrm{m}$.
\end{abstract}




\begin{tabular}{ccc|ccc}
\hline Model & angle, $\delta\left(^{\circ}\right)$ & span, $t(\mathrm{~mm})$ & Model & angle, $\delta\left(^{\circ}\right)$ & span, $t(\mathrm{~mm})$ \\
\hline W2510 & 25 & 10 & $\mathrm{~W} 4010$ & 40 & 10 \\
W2515 & 25 & 15 & $\mathrm{~W} 4015$ & 40 & 15 \\
W2520 & 25 & 20 & $\mathrm{~W} 4020$ & 40 & 20 \\
W2530 & 25 & 30 & $\mathrm{~W} 4030$ & 40 & 30 \\
W2540 & 25 & 40 & $\mathrm{~W} 4040$ & 40 & 40 \\
\hline
\end{tabular}

Table 1 Wedge model configurations

\section{Supersonic/Hypersonic Flow Past Finite-Span Wedge Fairings}

The supersonic/hypersonic flow about wedge-like fairings on the side of cylindrical bodies, where the wedge width is much smaller than body diameter, is approximately equivalent to that of the flow past finite span wedges mounted on a flat plate. This class of flow has been studied in the past [1-8] and involves a complex interaction between the wedge shock wave and the oncoming boundary layer, which can separate ahead of the wedge, generating a shock induced bow vortex, depending on the boundary layer state and the wedge angle. The flow will also feature separations from the sharp edges of the wedge which result in vortices being shed (Fig. 2). Here the upstream separation causes an effective shock wave detachment (separation shock), lambda shock interactions, and a large shock-induced bow vortex.
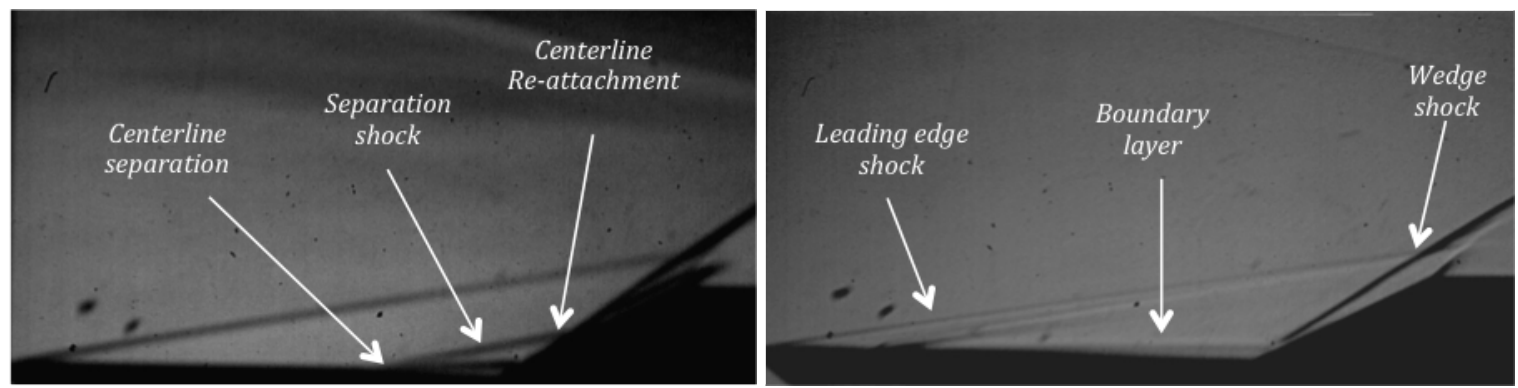

Fig. 1 Experimental Schlieren images of model W2540 in laminar (left) and turbulent (right) incoming flow conditions at $M=8.2$.

If the wedge is wide enough, the flow experienced by the forward face will correspond with that seen with the supersonic flow past a 2D ramp compression corner, which has been extensively studied [9-16]. Figure1 presents the flow structure typically seen in the interaction of an oncoming boundary layer with a ramp compression corner, where the adverse pressure gradient imposed by the ramp deflection causes an upstream separation and an associated separation shock wave and underlying separation bubble, together with a second shock wave associated with reattachment of the separated shear layer. This lambda shock wave pattern is characteristic of a hypersonic shock wave/boundary layer interaction.

A typical surface pressure distribution is presented in Fig. 4, which is characterised by the appearance of a pressure plateau under the separation bubble, and a pressure overshoot, beyond the level expected of a theoretical inviscid flow, which occurs due to the appearance of the reattachment shock wave.

This paper reports on an investigation of this class of flow, with a view to assess whether a combination of simple inviscid theory, coupled with experimentally derived estimations of the incipient wedge separation angle and upstream separation bubble characteristics can form the basis for an effective drag estimation method for finite span wedges in supersonic/hypersonic flows.

\section{Theoretical Modeling}

It is proposed that a simple drag estimation method for a wide range of finite span wedge fairing geometries in supersonic/hypersonic flows can be derived by the use of simple inviscid theory, simple surface friction prediction methods, and empirically derived relations for the separation bubble characteristic. If the effects of flow three- 


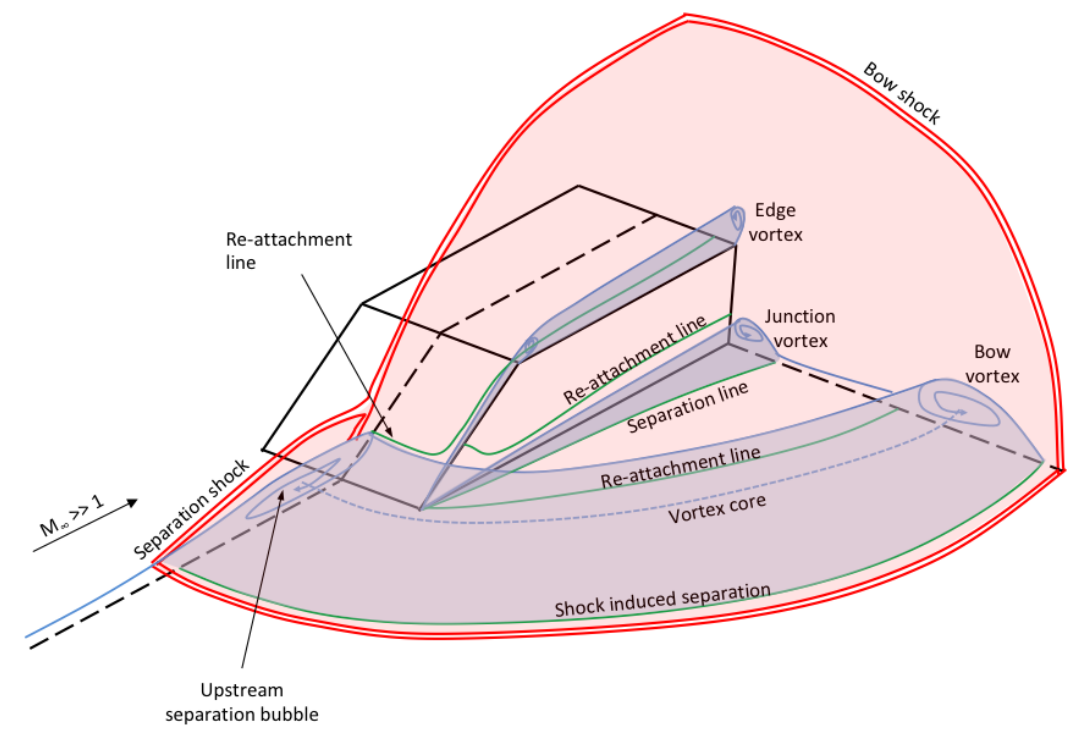

Fig. 2 Schematic of the typical three-dimensional flow features observed with supersonic flow past finite span wedges.

dimensionality, as the flow field progressively departs from that expected of a purely $2 \mathrm{D}$ interaction, can be described in a simple relation, a practical drag prediction model may be developed which can be used for a wide range of wedge geometries.

Figure 4 presents a simplified model for the 2D flow structure of interest, together with the surface pressure distribution typically observed, and a simplified theoretical model. The theoretical model assumes an instantaneous jump in surface pressure at the upstream boundary-layer separation location, a pressure plateau up to the wedge foot, and a linear rise in pressure up to the theoretical 2D inviscid post-shock level at the reattachment point, with a constant pressure beyond this, up to the top edge of the forward wedge face.

For the case of an attached boundary layer flow the theoretical surface pressure would rise instantaneously at the wedge corner, from the flat plate level to the theoretical 2D inviscid post-shock level.

Figure 3 presents the surface numbering convention used in this study, whereby the pressure and skin friction forces are estimated for each surface such that the total drag force on the fairing can be obtained from the addition of the contribution from each one.

\section{A. Pressure Distribution on the Forward Wedge Surface (S1)}

A model based on the blast wave analogy to account the 3D nature of the bow shock in cases where this is detached from the wedge apex was developed. Here the pressure force on the forward wedge surface was estimated using a number of theoretical approaches. The method of Boger \& Aiello [17] was used to predict the surface pressure level. This method is based on the theory of Cheng et al. (CT) [18], combining the 3D effect of nose bluntness (blast wave theory) and the boundary layer displacement (strong interaction theory).

If the effects of the three-dimensionality of the flow is neglected, shock-expansion theory (SE) and hypersonic slender body theory (HSB) can be employed to obtain the inviscid pressure level. The 2D hypersonic slender body relation for pressure coefficient for a given flow deflection, $\delta$ (in radians), is given by: 


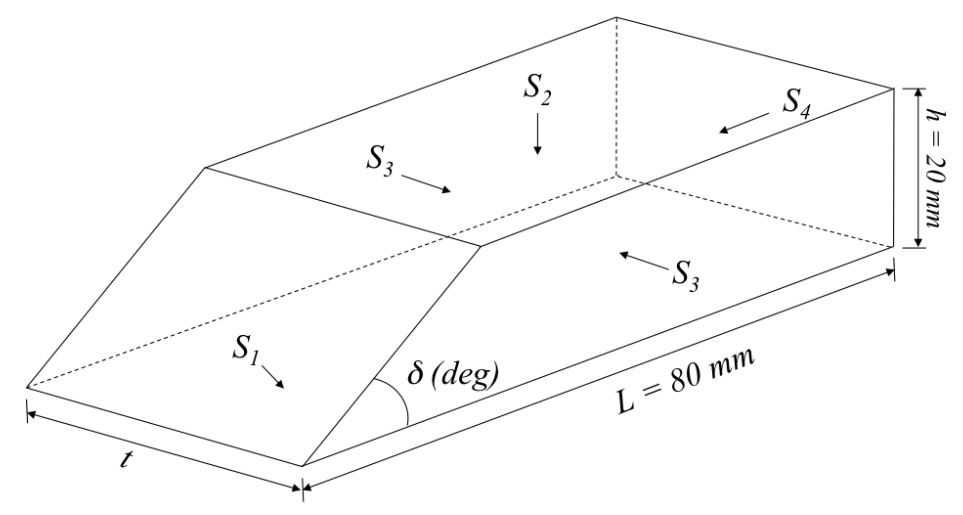

Fig. 3 Wedge surface numbering convention

\begin{tabular}{|l|l|}
\hline$C_{D, p}\left(S_{1}\right)$ & $C_{D, f}\left(S_{2}, S_{3}\right)$ \\
\hline Tangent-wedge (HSB) & Van Driest (VD) \\
Shock-expansion (SE) & Eckert (E) \\
Cheng (CT) & \\
\hline
\end{tabular}

Table 2 Combinations of drag force theories investigated

$$
\frac{C_{p 1}}{2 \delta^{2}}=\frac{\gamma+1}{4} \pm \sqrt{\left(\frac{\gamma+1}{4}\right)^{2}+\frac{1}{(\beta \delta)^{2}}}
$$

where, $\beta=\sqrt{M_{\infty}^{2}-1}$. In all three cases the pressure level in the vicinity of the upstream separation bubble is estimated by use of the following empirical relations:

$$
\begin{aligned}
C_{p 1} & =3 \sqrt{C_{f(x s)}} M_{\infty}^{-3 / 4} \\
C_{p 1} & =\frac{1.91\left(M_{\infty}^{2}-1\right)^{-0.309}}{R e_{x s}^{0.1}}
\end{aligned}
$$

All three of these theoretical approaches were used and compared with the experimental drag measurements to assess their practical validity for this class of flow. The skin friction contribution on the inclined wedge surface, S1 was neglected for separated flow cases as this was expected to be relatively much smaller than that on the side surfaces $\mathrm{S} 2$ and S3. Only the component of the friction force in the streamwise direction is relevant in this case, and where a separation bubble exists this component will be negative below the reattachment line, and positive above it.

\section{B. Skin Friction Prediction Models for Surfaces S2 and S3}

Two methods were employed for the surface skin friction estimations. The first was the standard theoretical model for compressible boundary layers developed by van Driest [19, 20]. This method has been widely employed in industrial 

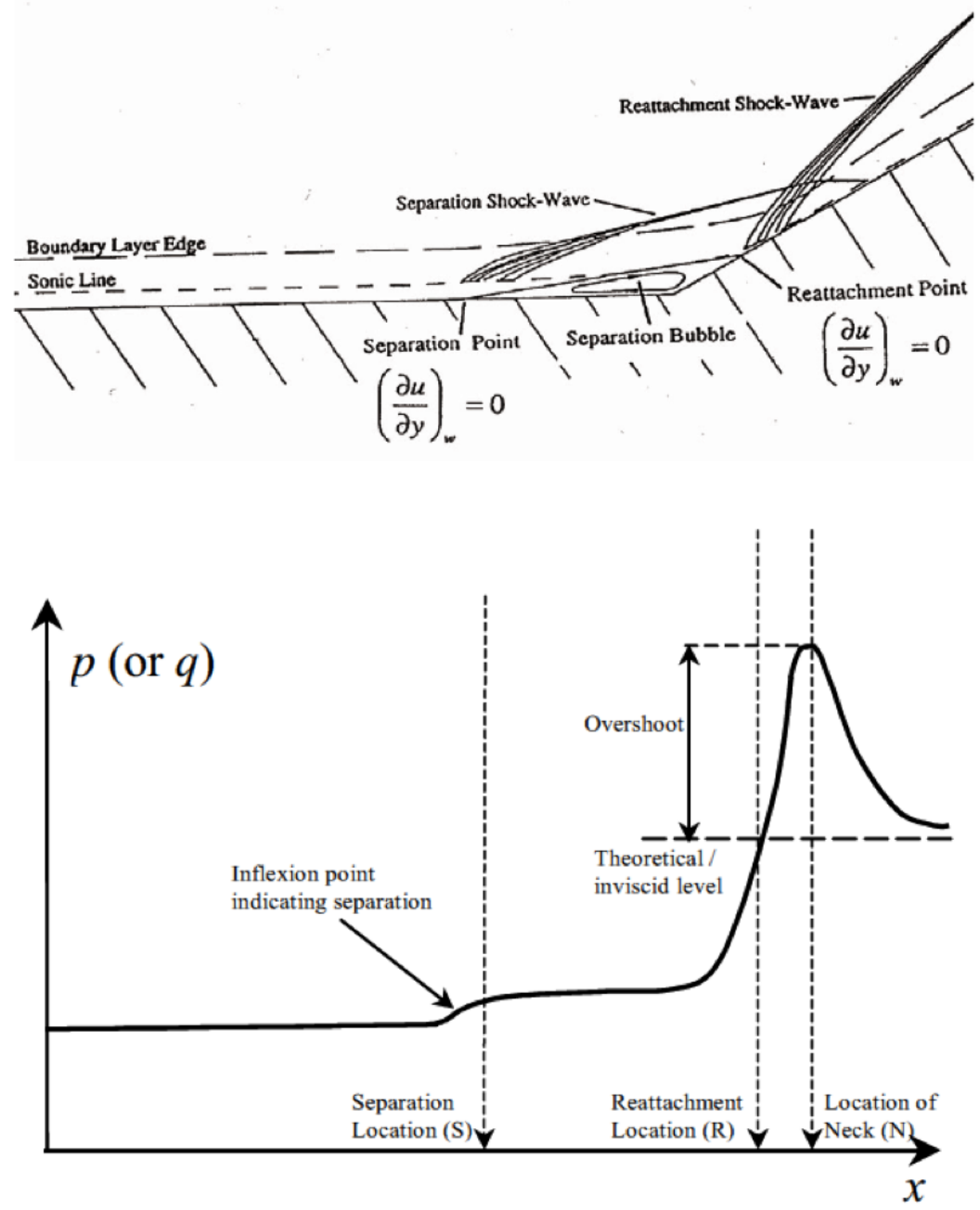

Fig. 4 Schematic of the shock wave-boundary layer interaction flow structure, and associated surface pressure variation for a ramp compression corner [12].

aerodynamics design codes. The other method assessed was that developed by Eckert [21], and based on the reference temperature concept. It is assumed that there is no contribution to the drag force of the wedge from the pressure acting on surfaces S2 and S3 for the case studied in this paper, of purely head-on freestream flow with no side-slip component. For cases with components of the flow velocity in the transverse axis (the z-axis in this case), the pressure contribution would need to be included in the same manner as has been done for the forward facing wedge surface.

\section{Base Pressure for Surface S4}

A significant contribution to the total drag of a wedge like fairing with a rear facing base surface is that due to the pressure on this surface, where the flow behind the fairing is likely to be fully separated. This base drag contribution is estimated in this analysis by employing the empirical derived equation for 3D base pressure of Love et al [22].

\section{Combinations for Total Drag Estimation}

Table 2 presents the six combinations of theories for the prediction of the total drag acting on the wedge fairing. These involve the addition of the pressure and skin friction contributions, obtained using one of the appropriate theories, for each surface. 


\section{Experimental Measurements}

Experimental measurements for the assessment and validation of the six theoretical models were obtained for a $\mathrm{M}=$ 8.2, Re $=9.0 \times 10^{6} / \mathrm{m}$, freestream flow in the Cranfield Hypersonic Gun Tunnel, which is an intermittent, free-piston, blow-down facility with a run time of about $40 \mathrm{~ms}[10,12,13]$.

The sting balance system measured three components of force/moments - normal and axial forces as well as pitching moment, with quoted accuracy of $\pm 5 \%$ at full scale. The Schlieren system was of a Z-pass design and employed a CREE Q5 LED light source, and a Photron APX high-speed camera which provided flow videos at $8000 \mathrm{fps}$ with a resolution of $1024 \times 256$ pixels.
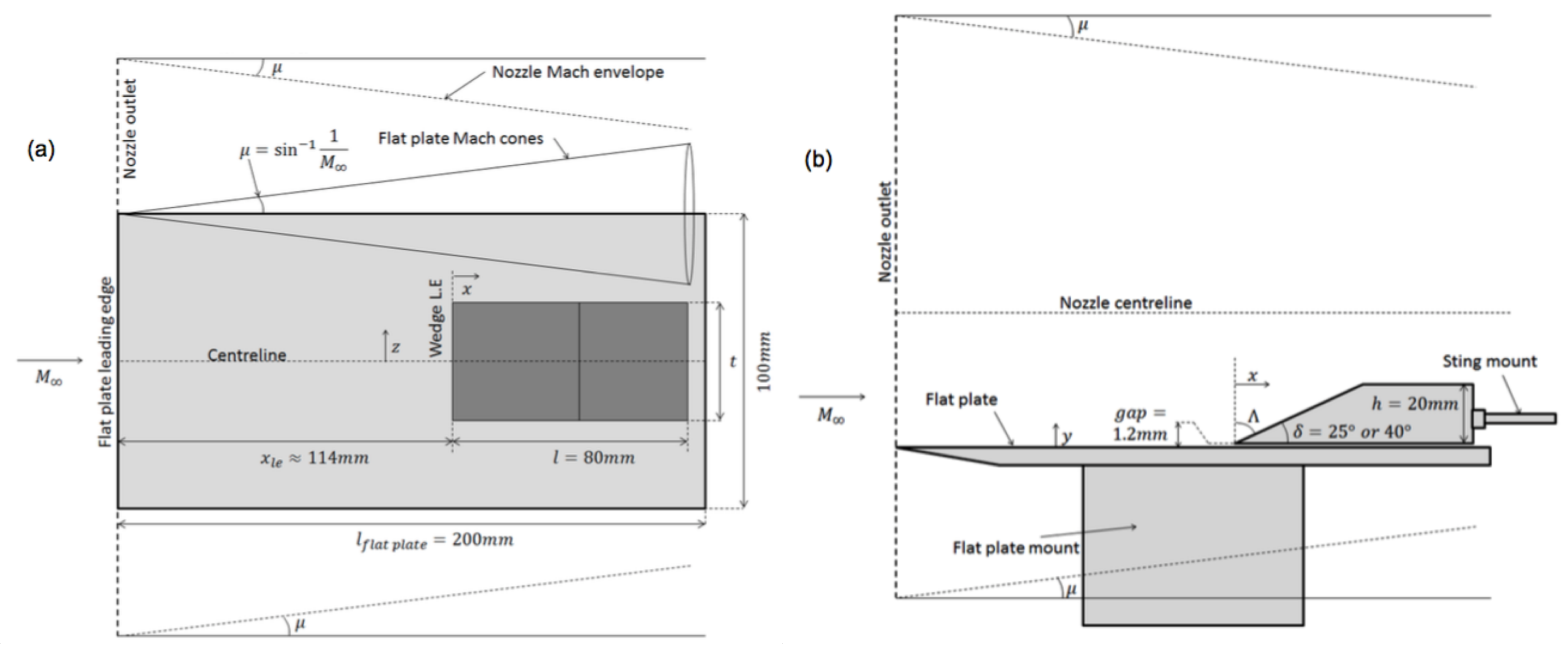

Fig. 5 Flat-plate/wedge fairing configuration within gun tunnel test section.

The experimental wedge models were mounted, via a rear connected sting balance system, $1.2 \mathrm{~mm}$ above a sharp leading edge flat plate model of $100 \mathrm{~mm}$ span and $200 \mathrm{~mm}$ length, as shown in Fig. 5. The wedge model was therefore isolated from the flat plate so that the sting balance measured only the forces acting on the model itself. A number of tests were done to assess the influence of the small gap between the model and the plate. If the gap was too large a significant through flow between the model and the plate develops, and the plate boundary layer/shock wave flow is no longer representative of the flow where no gap exists. If the gap is too small, the small deflections of the sting result in the model hitting the plate, thereby invalidating the drag measurements. The $1.2 \mathrm{~mm}$ gap was not found to influence the flow structure compared with the baseline flows imaged with the model firmly screwed to the plate.

The model was mounted in the working section as shown in Fig. 5, which presents both the geometric details as well as details of the nozzle and plate tip Mach cones for the $\mathrm{M}=8.2$ freestream, indicating the extent of downstream disturbances. The wedge model was located in a central position away from the plate edge effects.

Ten different wedge fairing configurations were tested, as listed in Table 1 Wedge angles of $25^{\circ}$ (below the incipient separation wedge angle for a turbulent interaction [15]) and $40^{\circ}$ were chosen, along with four span lengths, while the overall length and height of the model was kept constant. The principal dimension of interest in this study was therefore the span, or aspect ratio, of the wedge.

\section{Navier-Stokes CFD Simulations}

Along with the experimental test campaign, a numerical simulation study was also performed in order to i) assess whether modern CFD methods can adequately simulate this class of hypersonic flow and if so, ii) to provide more physical insight into this complicated flow.

For the numerical simulation study, the FLUENT commercial Navier-Stokes solver was employed. The flow was assumed to follow perfect gas behavior as the flow temperatures and densities were not expected to result in any significant molecular vibrational effects. Structured multiblock grids were used, whereby a grid sensitivity assessment 
was made with three progressively finer grids being tested, the finest having 3 million cells. A symmetry boundary condition was used on the plane passing through the z-centerline (through the center of the wedge span), while the flat plate sharp leading edge and side edges were modeled. Far-field flow domain box surfaces were located ten plate lengths away on all sides. The freestream boundary condition was set to match the gun tunnel flow properties during the period of the run when these were at their stabilized condition.

The first cell height on all wall surfaces was set at $1 \times 10^{-3} \mathrm{~mm}$, while the cell stretching was designed to ensure at least 20 cells within laminar (thinnest) boundary layers on the wedge surfaces. For the turbulent cases this gave $y+$ values of around 5 on the wedge surfaces. For this study the steady explicit/implicit Reynolds Averaged Navier-Stokes (RANS) solver was employed as the experimental flows observed did not appear to be highly unsteady. The solutions were found to converge well to a near steady-state result. However, the need to compute the unsteady flow is recognized, and this is being done as a follow-on study. Spalart-Allmaras, $k-\epsilon$ realized and $k-\omega$ SST turbulence models were tested and compared for all cases, and the latter model was found to provide the most accurate results, based on flow structure and averaged drag prediction. All turbulent CFD results presented here are, therefore, for the $k-\omega$ SST model.

The time averaged drag force coefficients were then calculated for comparison with the measured results and the theoretical predictions, while the centerline plane density gradient contours were compared with the experimental Schlieren photographs.

\section{Results}

\section{A. Experimental Flow Visualization}

Figure 1 presents a sample comparison of the Schlieren images obtained during the period of stabilized flow for the $25^{\circ}$ ramp angle, $40 \mathrm{~mm}$ span wedge with a) laminar and b) turbulent boundary layer flow. The upstream separation bubble is clearly seen in the laminar flow case, while the flow is seen to remain attached in the turbulent flow case. From these images, the separation and reattachment locations (on the centerline plane on which the features are seen) were then recorded and used for the theoretical drag predictions.

Figure 6 presents the variation, with wedge $l / t$, of the experimentally estimated separation bubble characteristics, and the relations derived to model these in the theoretical drag model. Figure 6 a shows that the location of upstream separation ahead of the wedge corner increases as the span of the wedge increases. Likewise, the overall length of the separated shear layer increases with increasing wedge span, as shown in Figure 6 b.

\section{B. Drag for Laminar Interactions}

The drag values predicted using Cheng's theory were found to consistently over-predict the drag force coefficient on the wedge, particularly for the low span cases where it gave drag predictions over twice as high as the measured results. A comparison of the results, against experiment, given by the six combinations of simple theories showed that the best predictions were obtained using SE+VD combination - shock-expansion theory (for the pressure drag) coupled with the van Driest method (for skin friction contribution), which was marginally better than using HSB+VD combination. The theoretical predictions presented here, are therefore only those obtained using the SE+VD combination, which is the one recommended for this application.

The variation with $l / t$ of the experimentally measured wedge drag coefficient, based on the wedge base cross-section area, is compared with the CFD computed and theoretically predicted values in figures 7 and 8 Also included are the estimated accuracy bars associated with the experimental measurements. Drag coefficient is seen to increase with reducing wedge span in all cases. Since, for a constant wedge angle, the ratio of the wedge frontal area and the base area remains constant with varying wedge span, as does the wedge side area, the only source for this change in drag coefficient is i) a variation in the size of the upstream separation bubble and ii) the effect of three-dimensional flow from the edges of the wedge.

Figure 7 present the comparison for the laminar flow cases where the overall trend in drag is correctly resolved by both the CFD model and the simple theoretical method. Agreement between measured and CFD computed is within 

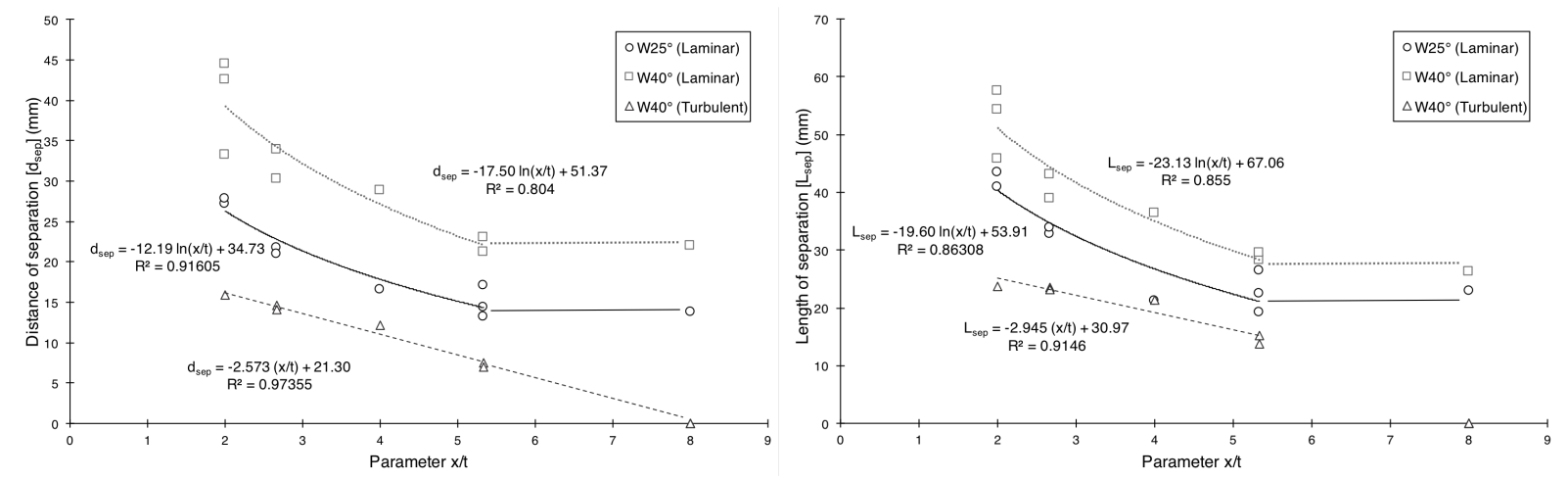

Fig. 6 Experimentally measured separation length ahead of the hinge, $d_{s e p}$, versus $l / t$ (left) and measured length of the separated shear layer, between separation and reattachment, $L_{s e p}$, versus $l / t$ (right)
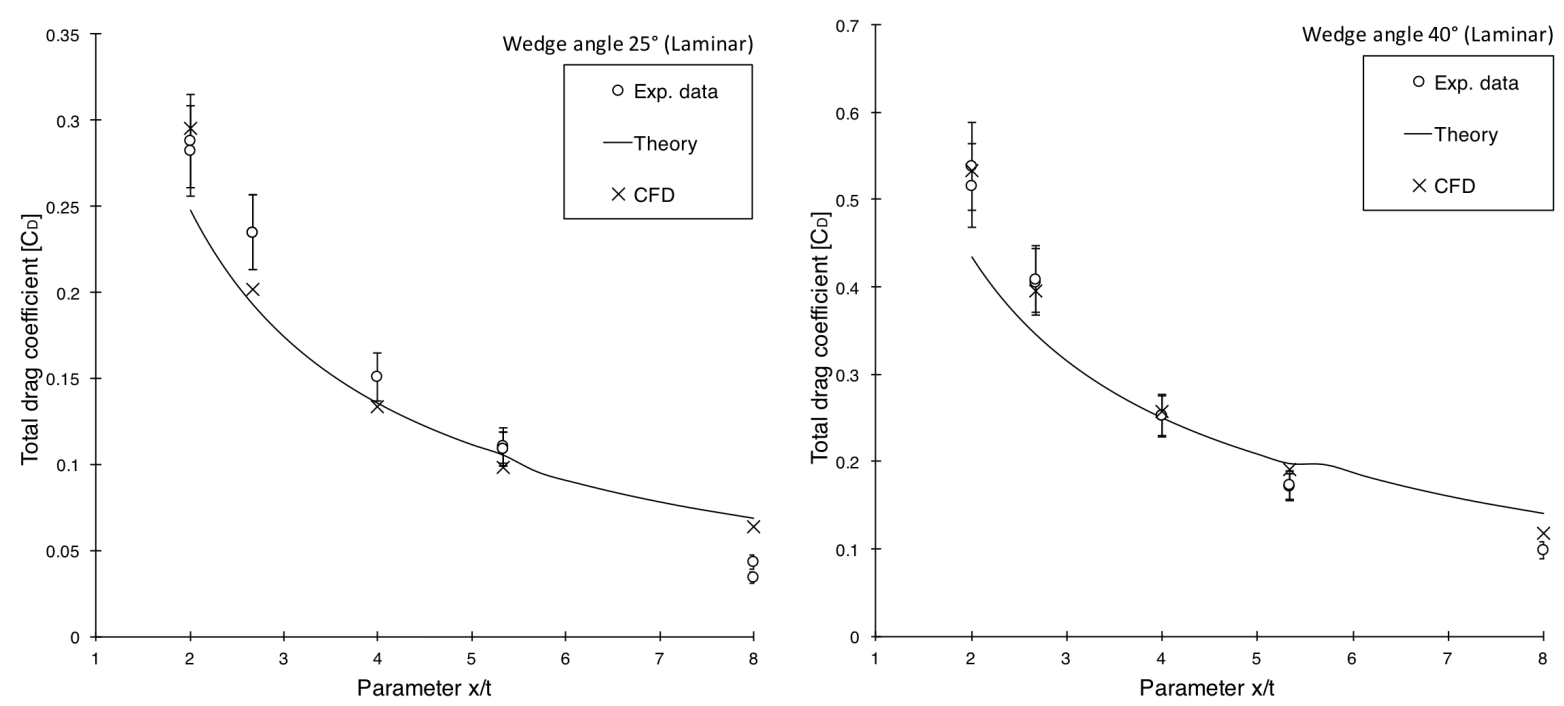

Fig. 7 Comparison of experimentally measured variation of drag coefficient with $l / t$, with theory and CFD under laminar interaction for $\delta=25^{\circ}$ (left) and $\delta=40^{\circ}$ (right).

$C_{D} \pm 0.03$, with much better agreement achieved for the $40^{\circ}$ wedge case. The theoretical model is seen to provide a reasonable estimation of the drag coefficient, on a par with the much more expensive CFD method and certainly within the requirements of a semi- empirical prediction method.

\section{Drag for Turbulent Interactions}

The same comparisons, but for the case of a turbulent interaction, are provided in Fig. 8. Here the agreement with the experimental measurements is much better for both the CFD and theoretical predictions. Both CFD and theory predicted drag coefficients are within $C_{D} \pm 0.03$ of the experimental measurements. This improved agreement is, in part, due to the higher levels of drag coefficient with turbulent incoming boundary layer.

The pressure level under the separation bubble, which is much larger for laminar incoming boundary layers, is very low thereby reducing the total pressure drag force on the wedge compared with a fully attached flow case. A turbulent boundary layer results in a much smaller separation bubble, and a much larger extent of high pressure on the wedge frontal face. 

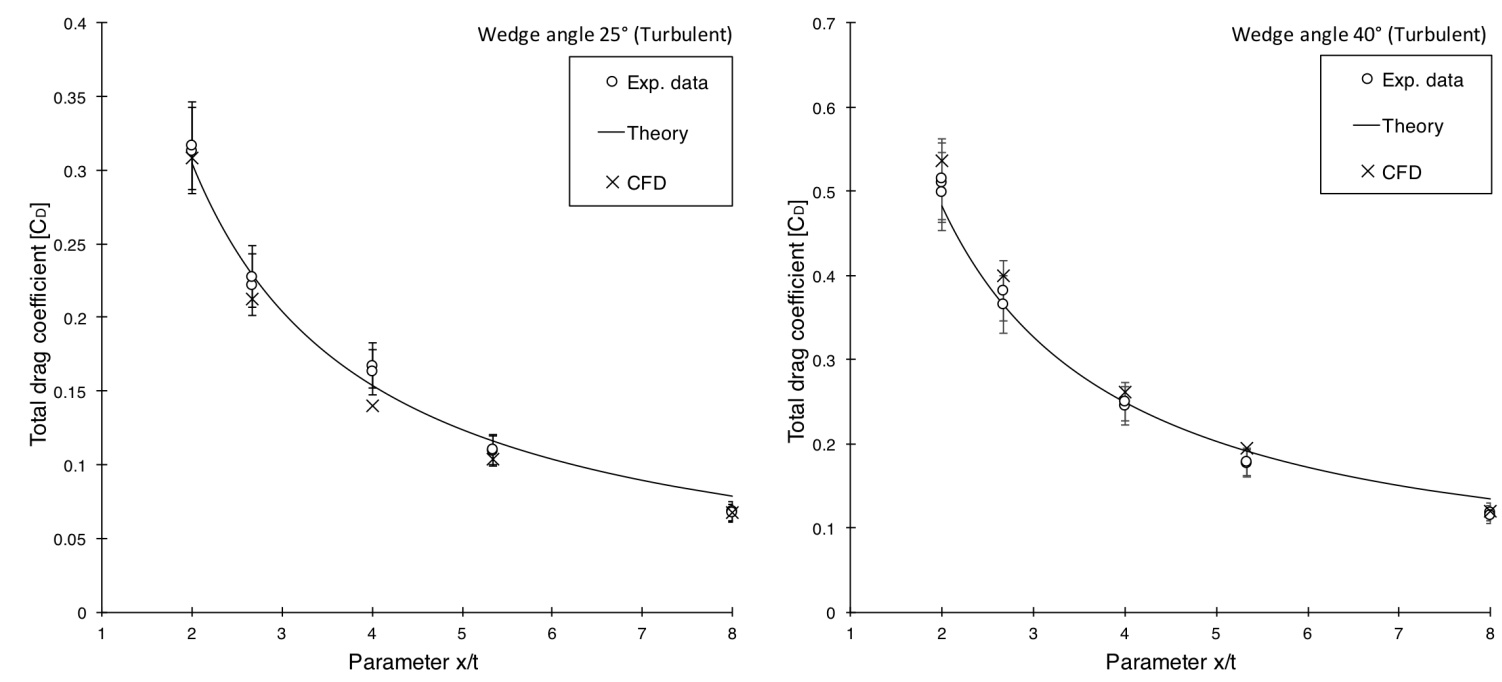

Fig. 8 Comparison of experimentally measured variation of drag coefficient with $l / t$, with theory and CFD under turbulent interaction for $\delta=25^{\circ}$ (left) and $\delta=40^{\circ}$ (right).

\section{Flow Structure - Insight from CFD Simulations}

The CFD solver is seen to have resolved both the structure and location of the major flow features very well. In particular the upstream separation bubble and its associated separation shock wave is seen to have been resolved accurately. The agreement between CFD and experiment tended to be better for the turbulent cases because the very large separation bubbles encountered with a laminar interaction were not as well captured in the CFD simulations.

The flow structure for an attached shock case and for a detached shock/upstream separation case, as resolved in the CFD simulations, are plotted for two cases in figure 9 . These images plot the contours of vorticity magnitude at an instant along with the surface skin friction lines on the flat plate. For the attached interaction case (model W2510, with turbulent plate boundary layer), shown in figure 9 , there is no upstream separation. The only separation is due to the formation of a vortex emanating from the wedge-plate corner. On the wedge, another pair of vortices are seen to form from the separations at the sharp edge between the side surface and wedge surface, which then grow as they expand onto the top surface of the wedge.

The flow structure for the detached shock wave case is shown in figure $9 \mathrm{~b}$, where the base of this detached bow shock forms a separation shock wave seen in the 2D Schlieren images. The flow structure in such cases is altogether more complex than that encountered with no shock detachment. Here the shock-induced separation line on the flat plate ahead of and around the sides of the wedge is clearly evident, as well as high levels of vorticity in the separation region ahead of the wedge.
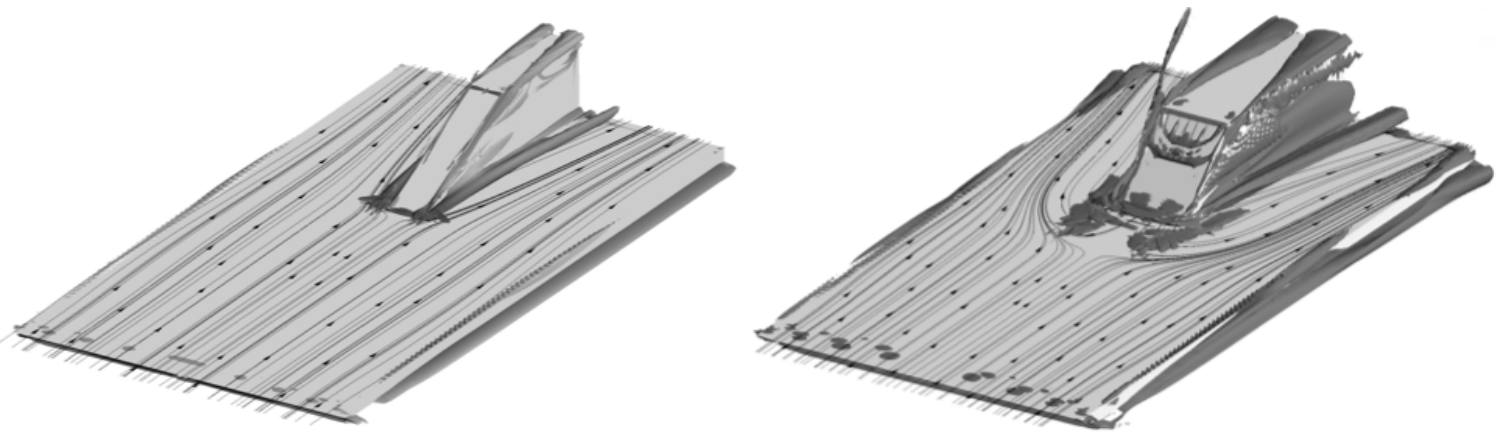

Fig. 9 CFD solution - contours of vorticity magnitude. Model W2510, Turbulent oncoming boundary layer (left) and model W4020, Laminar oncoming boundary layer (right) 
Also evident is the vorticity associated with the shear layer reattachment high up on the wedge surface. The corner junction vortex is still evident but this is seen to have been moved further outboard by the formation of a large bow vortex acting along the wedge side surfaces, which is associated with the primary shock- induced separation on the flat plate surface. This predicted flow structure is in agreement with those observed in previous studies, as was depicted in Fig. 2. except that the bow vortex is much further inboard in this particular case.

\section{Conclusion}

A semi-empirical model for the drag force acting on a wedge-like fairing in supersonic/hypersonic flow, mounted on a flat plate or a surface of relatively low curvature, has been developed. This has been validated for the case of a $M=8.2$ flow with both a laminar and a turbulent oncoming boundary layer state.

Much further work needs to be done to assess the method for a wide range of Mach numbers and wedge shapes, before it is ready for general use in the estimation of full configuration drag, but the results of this early study provide some confidence that a simple and relatively accurate general method of this kind is feasible.

\section{References}

[1] Burbank, P. B., Collins, I. K., and Newlander, R. A., "Heat-transfer and pressure measurements on a flat-plate surface and heat-transfer measurements on attached protuberances in a supersonic turbulent boundary layer at Mach numbers of 2.65, 3.51, and 4.44," 1962.

[2] Hung, F., and Patel, D., "Protuberance interference heating in high-speed flow," 19th Thermophysics Conference, 1984, p. 1724.

[3] Estruch, D., Lawson, N. J., MacManus, D. G., Garry, K. P., and Stollery, J., "Measurement of shock wave unsteadiness using a high-speed schlieren system and digital image processing," Review of Scientific Instruments, Vol. 79, No. 12, 2008, p. 126108.

[4] Estruch-Samper, D., Hypersonic interference aerothermodynamics, Cranfield University (United Kingdom), 2009.

[5] Estruch, D., MacManus, D. G., Stollery, J., Lawson, N. J., and Garry, K. P., "Hypersonic interference heating in the vicinity of surface protuberances," Experiments in Fluids, Vol. 49, No. 3, 2010, pp. 683-699.

[6] Le Rallic, M., "Effect of nose shaped protuberances in supersonic flow,” Master's thesis, Cranfield University, 2012.

[7] Cooper, A., "Effect of span on supersonic flowpast a wedge shaped protuberance," Master's thesis, Cranfield University, 2012.

[8] Roussel, A., Prince, S., Viguer, M., Kshitij, A., Stollery, J., and Garry, K., “The Development of a Simple Method for Drag Estimation for Wedge-Like Fairings in Hypersonic Flow," ????

[9] Bogdonoff, S., "Separation of a supersonic turbulent boundary layer," Journal of the Aeronautical Sciences, Vol. 22, No. 6, 1955, pp. 414-430.

[10] Needham, D., and Stollery, J., "Boundary layer separation in hypersonic flow," 3rd and 4th Aerospace Sciences Meeting, 1966, p. 455.

[11] Popinski, Z., and Ehrlich, C., Development design methods for predicting hypersonic aerodynamic control characteristics, Air Force Flight Dynamics Laboratory, Research and Technology Division, Air ..., 1966.

[12] Elfstrom, G., "Turbulent hypersonic flow at a wedge-compression corner," Journal of fluid Mechanics, Vol. 53, No. 1, 1972, pp. 113-127.

[13] Coleman, G. T., "Hypersonic turbulent boundary layer studies," Ph.D. thesis, Imperial College, London, 1973.

[14] Hankey Jr, W., and Holden, M. S., "Two-dimensional shock wave-boundary layer interactions in high speed flows,” Tech. rep., AGARD, 1975.

[15] Kumar, D., "Hypersonic Control Effectiveness," Ph.D. thesis, Cranfield University, 1995.

[16] Prince, S., Vannahme, M., and Stollery, J., "Experiments on the hypersonic turbulent shock-wave/boundary-layer interaction and the effects of surface roughness," The Aeronautical Journal, Vol. 109, No. 1094, 2005, pp. 177-184. 
[17] Boger, R., and Aiello, G., "Hypersonic flow on yawed wedges with leading-edge bluntness and viscous interaction," Journal of Spacecraft and Rockets, Vol. 8, No. 7, 1971, pp. 729-735.

[18] Cheng, H., "Boundary-layer displacement and leading-edge bluntness effects in high-temperature hypersonic flow," Journal of the Aerospace Sciences, Vol. 28, No. 5, 1961, pp. 353-381.

[19] Van Driest, E. R., "Turbulent boundary layer in compressible fluids," Journal of spacecraft and rockets, Vol. 40, No. 6, 2003, pp. 1012-1028.

[20] Van Driest, E., "Investigation of laminar boundary layer in compressible fluids using the Crocco method. NACA Tech,” Tech. rep., Note 2597, 1952.

[21] Eckert, E., "Engineering relations for friction and heat transfer to surfaces in high velocity flow," , 1955.

[22] Love, E. S., "The Base Pressure at Supersonic Speeds on Two-Dimensional Airfoils and Bodies of Revolution (With and Without Fins) Having Turbulent Boundary Layers," 1953. 
2020-01-05

\title{
Drag estimation on wedge-shaped protuberances in high-speed flows
}

\author{
Kshitij, Abhinav
}

AIAA

Kshitij A, Prince SA, Stollery JL. (2020) Drag estimation on wedge-shaped protuberances in high-speed flows. In: 2020 AIAA SciTech Forum, 6-10 January 2020, Orlando, Florida, USA https://doi.org/10.2514/6.2020-2053

Downloaded from Cranfield Library Services E-Repository 